Francis R. Verdun

D. Lepori

Pascal Monnin

Jean-François Valley

Pierre Schnyder

F. Gudinchet

\section{Management of patient dose and image noise in routine pediatric CT abdominal examinations}

Received: 18 July 2003

Revised: 5 November 2003

Accepted: 1 December 2003

Published online: 13 January 2004

(C) Springer-Verlag 2004
F. R. Verdun ( $)$ P. Monnin · J.-F. Valley University Institute

for Applied Radiophysics,

Grand-Pré 1, 1007 Lausanne, Switzerland

e-mail: francis.verdun@hospvd.ch

Tel.: +41-21-6233434

Fax: +41-21-6233435

D. Lepori · P. Schnyder · F. Gudinchet

Department of Diagnostic

and Interventional Radiology,

University Hospital Center (CHUV),

1011 Lausanne, Switzerland

\begin{abstract}
The aim was to propose a strategy for finding reasonable compromises between image noise and dose as a function of patient weight. Weighted CT dose index $\left(\mathrm{CTDI}_{\mathrm{w}}\right)$ was measured on a multidetector-row CT unit using CTDI test objects of 16,24 and $32 \mathrm{~cm}$ in diameter at 80 , 100,120 and $140 \mathrm{kV}$. These test objects were then scanned in helical mode using a wide range of tube currents and voltages with a reconstructed slice thickness of $5 \mathrm{~mm}$. For each set of acquisition parameter image noise was measured and the Rose model observer was used to test two strategies for proposing a reasonable compromise between dose and lowcontrast detection performance:
\end{abstract}

(1) the use of a unique noise level for all test object diameters, and (2) the use of a unique dose efficacy level defined as the noise reduction per unit dose. Published data were used to define four weight classes and an acquisition protocol was proposed for each class. The protocols have been applied in clinical routine for more than one year. $\mathrm{CTDI}_{\mathrm{vol}}$ values of 6.7, 9.4, 15.9 and $24.5 \mathrm{mGy}$ were proposed for the following weight classes: $2.5-5,5-15,15-30$ and $30-50 \mathrm{~kg}$ with image noise levels in the range of 10-15 HU. The proposed method allows patient dose and image noise to be controlled in such a way that dose reduction does not impair the detection of low-contrast lesions. The proposed values correspond to highquality images and can be reduced if only high-contrast organs are assessed.

Keywords Pediatric CT .

Dose reduction - Dosimetry .

Image quality $\cdot$ Optimization

\section{Introduction}

CT examinations represent about $2-6 \%$ of all radiological examinations performed on adults, but contribute $27-40 \%$ of the effective dose delivered in radiology [1, $2,3,4]$. In this context, several strategies have been adopted by manufacturers to reduce patient dose $[5,6$, $7,8]$, and the many papers devoted to CT dose assessment have increased radiologists' awareness of the high dose level associated with CT examinations $[9,10,11$, 12]. The use of pediatric $\mathrm{CT}$ is increasing quite rapidly, probably more rapidly than for adult $\mathrm{CT}$, even if not used as a first-line imaging modality in many cases. It is an important diagnostic tool that certainly improves health care. However, the technical parameters chosen for an examination must be such that radiation risks are maintained as low as reasonably achievable for the diagnostic intent. For a given set of machine parameters (voltage, tube current-time product, pitch, etc.), it has been shown that effective doses for a small infant are larger than for an adult $[13,14,15]$. As a result, pediatric CT acquisition protocols need to be carefully controlled.

Several approaches have been used to propose image acquisition parameters that give a reasonable compromise between image quality and patient dose, some based on the assessment of clinical data $[15,16,17,18]$ and others on simulated data $[19,20,21]$. However, while these studies show that it is possible to reduce the 
dose for pediatric CT, there is still a need to define objectively levels of image quality adequacy.

The goal of this paper is to propose a general method for setting dose levels for abdominal pediatric CT acquisitions using CTDI test objects. We consider the CT unit from a dose point of view, and also in terms of the adequacy of the acquisition parameters for detecting lowcontrast lesions using a mathematical model observer.

\section{Materials and methods}

Dose characterization of the unit

The Multi-detector row CT (MDCT) used for this study was a fourrow LightSpeed QX/I (GE Medical Systems, Milwaukee, Wis). Acquisitions were performed with a detector collimation of $2.5 \mathrm{~mm}$, acquiring four slices per $360^{\circ}$ tube rotation. For each tube voltage $(\mathrm{kV})$, the normalized weighted CTDI, ${ }_{n} \mathrm{CTDI}_{\mathrm{w}}$, (mGy mAs${ }^{-1}$ ), was measured using the standard PMMA CTDI test objects of 16 and $32 \mathrm{~cm}$ in diameter [22]. Measurements of ${ }_{n} \mathrm{CTDI}_{\mathrm{w}}$ were also made in air at the center of rotation of the gantry and in a PMMA CTDI test object of $24 \mathrm{~cm}$ in diameter designed for this study. Doses delivered during helical acquisitions were

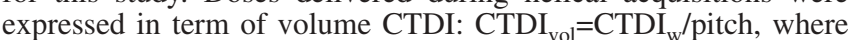
pitch is defined as the table distance traveled in one rotation of the $\mathrm{X}$-ray source divided by the total collimated width of the X-ray beam $[22,23]$. A subscript was added to the standard notation to indicate the test object diameter in which the measurement was expressed (e.g., a CTDI ${ }_{\text {vol }}$ measured in a CTDI test object of $16 \mathrm{~cm}$ diameter is quoted as $\left.\mathrm{CTDI}_{\mathrm{vol}, 16}\right)$.

\section{Image noise characterization}

The three CTDI test objects were imaged in helical mode with a pitch of 0.75 , detector collimation of $2.5 \mathrm{~mm}$ (i.e., $4 \times 2.5 \mathrm{~mm}$ ), reconstructed slice thickness of $5 \mathrm{~mm}$, and at tube voltages of 80 , 100,120 and $140 \mathrm{kV}$ for a wide range of tube currents (i.e., $40-400 \mathrm{~mA}$ in steps of $40 \mathrm{~mA}$ ) using a $360^{\circ}$ tube rotation time of $0.8 \mathrm{~s}$. The detector collimation and reconstructed slice thickness chosen correspond to the acquisition conditions used for routine pediatric abdominal examinations in our center. For each acquisition, the reconstructed field of view was set equal to $4 \mathrm{~cm}$ larger than the diameter of the imaged CTDI test object. To assess image noise, the standard deviation of CT numbers was measured in a 300-pixel region of interest (ROI) at five locations of the test object (one measurement at the center and four measurements at $1 \mathrm{~cm}$ from the periphery of the test object) and an average standard deviation, $\sigma$, was calculated. Measurements were performed on one image for each combination of test object and CTDI ${ }_{\mathrm{vol}}$ values using the software available on the CT unit.

\section{Compromise between noise and image noise}

One possible way to set reasonable dose levels is to choose an image noise level and keep it constant whatever the abdominal diameter of the patient. Another possibility is to look at the rate at which noise decreases as dose increases, as a function of the abdominal diameter of the patient. From the statistics properties of quantum noise (i.e., image noise inversely proportional to the square root of dose) it appears that at some point as dose increases we start to lose in the strides we make in noise reduction. Thus, there is a dose level above which noise reduction with dose increase is no longer justified. To determine the adequacy of the im-

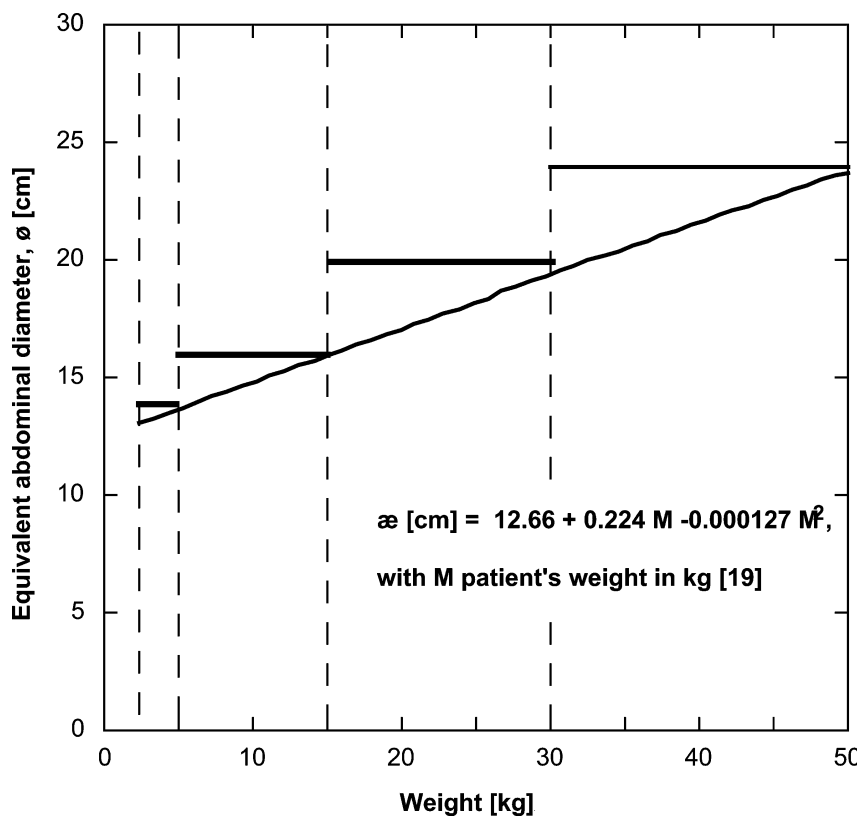

Fig. 1 Equivalent abdominal diameter as a function of patient weight according to Huda et al. [19]

age acquisition protocol, minimum image quality requirements were set by specifying the size and contrast of the structure to be detected. Only acquisition protocols allowing the detection of a low-contrast structure (i.e., having a contrast with background equal to $10 \mathrm{HU}$ whatever the tube voltage used) smaller than or equal to $10 \mathrm{~mm}$ were accepted. This choice leads to comparable low-contrast detection requirements as the ones tested, for example, when using standard CT test objects such as the Catphan 500 (The Phantom Laboratory, Cambridge, NY) and thus assures a good level of image quality. To establish whether the acquisition protocols fulfilled the low-contrast detection constraint proposed, the Rose model observer was applied on each combination of test object and $\mathrm{CTDI}_{\mathrm{vol}}$ values. This model is described by the following relationship: $S N R=C N R \times(N)^{1 / 2}$, where CNR is the contrastto-noise ratio (i.e., $10 \mathrm{HU}$ divided by averaged standard deviation, $\sigma$ ) and $N$ the number of pixels of the low-contrast structure to be detected. For structure diameter $d$ and pixel size dimension $\Delta, N$ is given by; $(\pi / 4) \times(d / \Delta)^{2}$ [24] (see Table 2). A SNR value of 5.0 was systematically adopted when using this model observer since it corresponds to a situation where detection is performed with a high confidence level.

Link between experimental results and clinical applications

To relate the experimental results to clinical applications, patient weights were converted to equivalent diameter of the abdominal section using the relationship proposed by Huda et al. [19] illustrated in Fig. 1. To reduce the number of acquisition protocols, the pediatric population was split into four weight classes: 2.5-5, $5-20,20-35$, and $35-50 \mathrm{~kg}$, giving the following equivalent diameters: $14,16,20$, and $24 \mathrm{~cm}$.

\section{Clinical application}

The resulting protocols were applied in routine use for one year (507 standard abdominal examinations). For ethical reasons, ac- 
Table 1 Fit parameters of the relationships between the ${ }_{n} \mathrm{CTDI}_{\mathrm{w}}$ parameter and the diameter of the CTDI test object used to perform the measurements: ${ }_{n} C T D I_{w}=$ a test object $\emptyset+b$

\begin{tabular}{rlll}
\hline $\begin{array}{l}\text { Tube voltage } \\
(\mathrm{kV})\end{array}$ & $a\left(\mathrm{mGy} \mathrm{mAs}^{-1} \mathrm{~cm}^{-1}\right)$ & $b\left(\mathrm{mGy} \mathrm{mAs}^{-1}\right)$ & $\begin{array}{l}\text { Regression } \\
\text { coefficient }\end{array}$ \\
\hline 80 & -0.00223 & 0.103 & 0.998 \\
100 & -0.00365 & 0.182 & 0.999 \\
120 & -0.00555 & 0.282 & 0.999 \\
140 & -0.00789 & 0.401 & 0.999 \\
\hline
\end{tabular}

quisition protocols while varying dose levels were not tested. The use of the model observer was considered sufficient to assure that image noise were sufficiently low to provide images of good diagnostic quality. All cases were reviewed and evaluated with consensus by three senior radiologists (FG, DL, PS). The adequacy of image quality was determined by asking the radiologists to assess the perceived level of mottle in the CT images and to assign each examination to one of two categories:

- Inadequate for diagnosis

- Diagnostically adequate

\section{Results}

For each tube voltage, a linear relationship could be established between the ${ }_{n} \mathrm{CTDI}_{\mathrm{w}}$ and the test object diameter with a regression coefficient higher than 0.990 (see Table 1). The measured $\mathrm{CTDI}_{\mathrm{vol}}$ with the test objects of 16 and $32 \mathrm{~cm}$ in diameter were systematically in good agreement with the values indicated by the unit (differences within $\pm 10 \%$ ).

For each image acquisition condition, the mean CT number measured in each of the four ROIs was within the averaged standard deviation, $\sigma$. The relationship between the averaged standard deviation values, $\sigma$, and the volume CTDI, CTDI ${ }_{\mathrm{vol}, \varnothing}$, for the three test objects are presented in Fig. 2. These data shows that a low noise level can hardly be obtained when imaging large test objects. Figure 3 shows the derivatives, $E_{\mathrm{f}}$, with respect to dose, of the data presented in Fig. 2. The parameter $E_{\mathrm{f}}$ represents the noise reduction per $\mathrm{mGy}$ as a function of the $\mathrm{CTDI}_{\mathrm{vol}}$ and is a mean to evaluated the dose efficacy in reducing image noise. This graph shows that in the low dose range a small increase in dose allows a drastic noise reduction, whereas in the high dose range dose efficacy becomes very low.

The diameter of a structure with a contrast of $10 \mathrm{HU}$ with background which can be detected with a high confidence level according to the Rose model used in this study was then calculated. The results obtained for each test object are shown in Fig. 4. For each test object the dose efficacy levels (data presented in Fig. 3) of 0.25, 0.5 and 1.0 have been added on the graph. As expected, large differences in dose are needed to maintain the lowcontrast detection performance as the test object diameter is varied from $16 \mathrm{~cm}$ to $32 \mathrm{~cm}$.

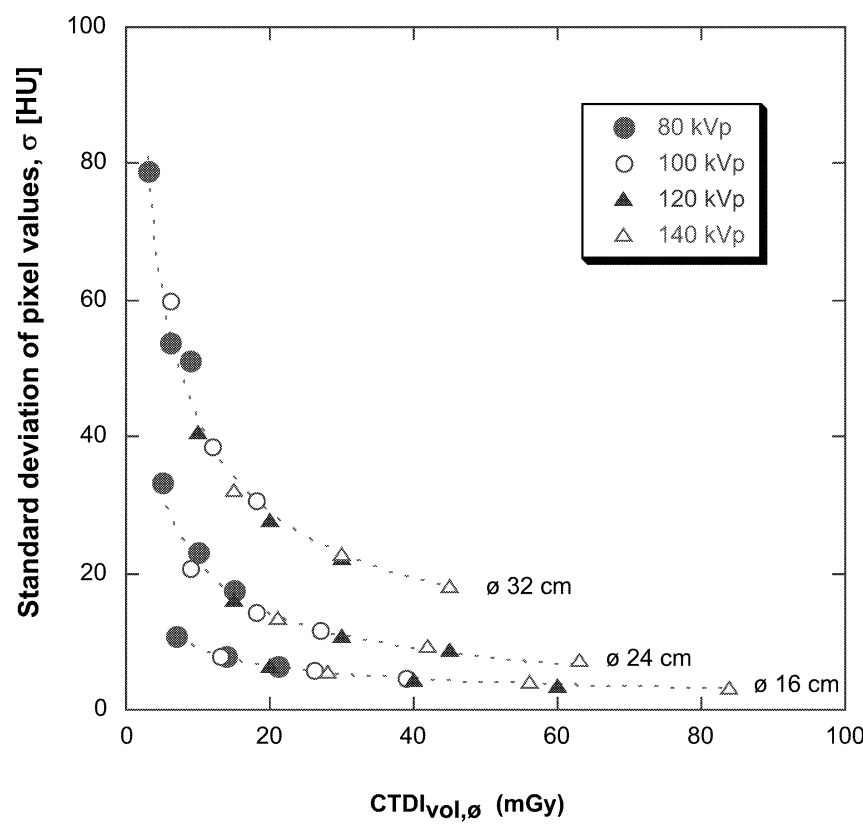

Fig. 2 Averaged standard deviation (i.e., image noise) variations as a function of $\mathrm{CTDI}_{\mathrm{vol}}$ in test objects of 16,24 and $32 \mathrm{~cm}$ diameter

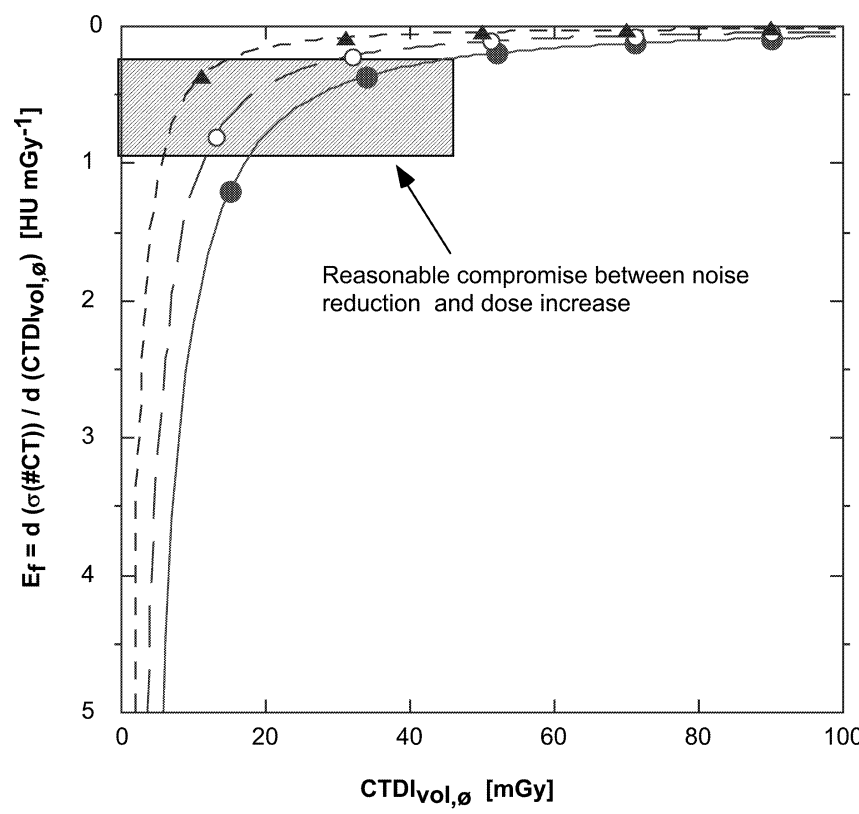

Fig. 3 Dose efficacy, $E_{\mathrm{f}}$, as a function of $\mathrm{CTDI}_{\mathrm{vol}, \varnothing}$ for 16,24 and $32 \mathrm{~cm}$ diameter test objects. As expected there exists a range of $\mathrm{CTDI}_{\mathrm{vol}, \varnothing}$ values where a modest increase in dose drastically reduces image noise. It can also be shown also that in the high $\mathrm{CTDI}_{\mathrm{vol}, \varnothing}$ range, the dose efficacy becomes very low. The hatchmarked region represents the position along the noise versus dose curve where a reasonable compromise between image quality and patient dose is expected 
Table 2 Smallest diameters, $d$, of a structure having a contrast with background of $10 \mathrm{HU}$, detectable according to the Rose criteria as a function of the dose efficacy $E_{\mathrm{f}}$

\begin{tabular}{llcccc}
\hline $\begin{array}{l}E_{\mathrm{f}} \\
\left(\mathrm{HU} \mathrm{mGy}{ }^{-1}\right)\end{array}$ & $\begin{array}{l}\text { Test object } \\
\varnothing(\mathrm{cm})\end{array}$ & $\begin{array}{l}\mathrm{CTDI}_{\mathrm{vol}, \varnothing} \\
(\mathrm{mGy})\end{array}$ & $\sigma(\mathrm{HU})$ & $\Delta(\mathrm{mm})$ & $\mathrm{d}(\mathrm{mm})$ \\
\hline 1.0 & 16 & 5.9 & 11.8 & 0.391 & 2.6 \\
1.0 & 24 & 11.7 & 19.8 & 0.547 & 6.1 \\
1.0 & 32 & 17.5 & 31.2 & 0.703 & 12.4 \\
0.5 & 16 & 9.4 & 9.4 & 0.391 & 2.1 \\
0.5 & 24 & 18.5 & 15.0 & 0.547 & 4.6 \\
0.5 & 32 & 27.8 & 24.0 & 0.703 & 9.5 \\
0.25 & 16 & 14.8 & 7.5 & 0.391 & 1.7 \\
0.25 & 24 & 29.4 & 11.2 & 0.547 & 3.5 \\
0.25 & 32 & 44.2 & 18.2 & 0.703 & 7.2 \\
\hline
\end{tabular}

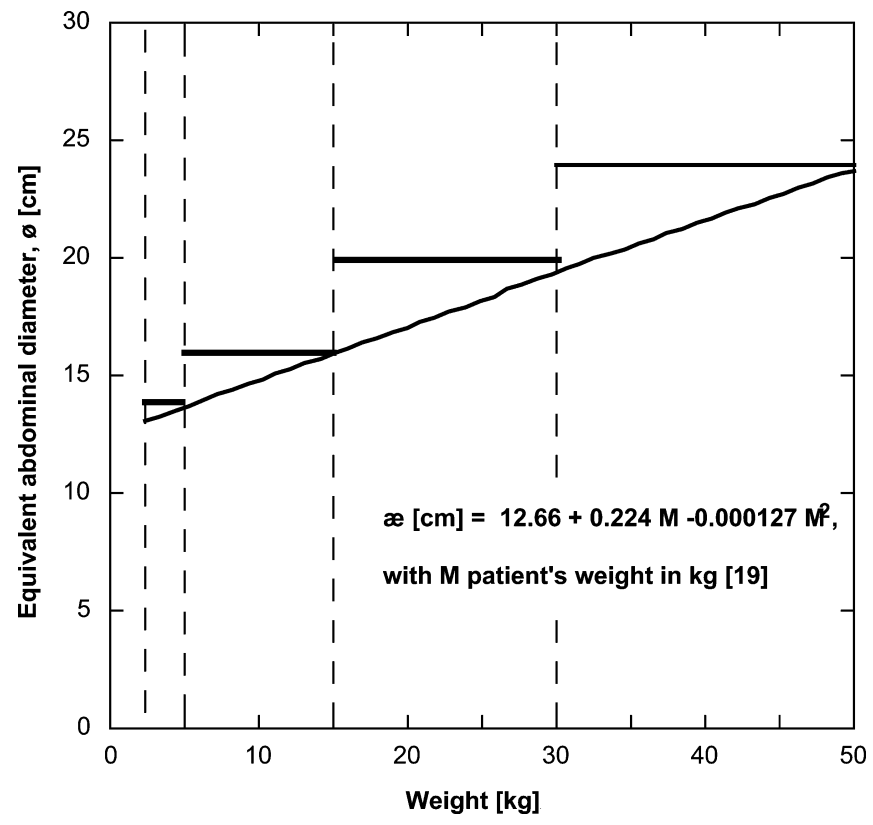

Fig. 4 Diameter of the smallest structure, $d$ (having a contrast with a background of $10 \mathrm{HU}$ ), detectable according to the Rose criteria as a function of $\mathrm{CTDI}_{\mathrm{vol}}$ for the three test objects involved in the study. The diameter, $d$, of the structure detectable with a high confidence level is also indicated for each test object at dose efficacy levels: $E_{\mathrm{f}}=1.0,0.5$ and 0.25

First option: use of a fixed image noise level

Using the data from Fig. 4 it is possible to choose an image quality level in term of low-contrast detection and to keep it constant, whatever the size of the CTDI test object. For example, the detection of a $10-\mathrm{mm}$ structure requires a CTDI ${ }_{\mathrm{vol}, 16}$ of $0.4 \mathrm{mGy}$, a CTDI ${ }_{\mathrm{vol}, 24}$ of $4.9 \mathrm{mGy}$, and a $\mathrm{CTDI}_{\mathrm{vol} .32}$ of $25.5 \mathrm{mGy}$ in 16-, 24- and $32-\mathrm{cm}-$ diameter test objects, respectively. This represents a factor of almost 64 in dose between the 16- and 32-cm-diameter test objects. If the size of the structure to be detected is decreased by a factor of 2 (i.e., $d=5 \mathrm{~mm}$ ), the dose is increased by factors in the range of 3.1-4.0 $\left(\mathrm{CTDI}_{\mathrm{vol}, 16}\right.$ of $1.6 \mathrm{mGy}, \mathrm{CTDI}_{\mathrm{vol}, 24}$ of $16.3 \mathrm{mGy}$ and $\mathrm{CTDI}_{\mathrm{vol}, 32}$ of $79.5 \mathrm{mGy}$ ). Thus, significant increases in dose are required with increasing diameter if a constant low-contrast detection performance requirement is applied to all test object diameters. Consequently, this strategy seems inadequate when dealing with large variations in test object or patient diameter.

Second option: use of a fixed dose efficacy

An alternative method for finding a reasonable compromise between patient dose and image noise is to choose a dose efficacy level, $E_{\mathrm{f}}$, and to estimate the $\mathrm{CTDI}_{\mathrm{vol}}$ that corresponds to that level. From Figs. 3 and 4 it appears that an $E_{\mathrm{f}}$ value in the range of $0.25-1.0 \mathrm{HU} \mathrm{mGy}^{-1}$ offers a good compromise between low-contrast detection and dose. The standard deviation and size of the smallest lesion detectable in this range of dose efficacy are summarized in Table 2. From these data it can be seen that a $10-\mathrm{mm}$ low-contrast structure can be detected in all of the test objects with a dose efficacy value of $0.5 \mathrm{HU}$ $\mathrm{mGy}^{-1}$. This value was chosen to define the acquisition protocols to be used in routine clinical practice.

\section{Clinical use}

Using the diameter of children as a function of age and making interpolation of the $\mathrm{CTDI}_{\mathrm{vol} . \varnothing}$ and image noise, $\sigma$, with a dose efficacy of 0.5 , the following $\mathrm{CTDI}_{\mathrm{vol}, \varnothing}$ values to be applied in clinical routine were found:

$$
\begin{aligned}
- & 2.5-5 \mathrm{~kg} \rightarrow \text { equivalent } \varnothing \text { of } 14 \mathrm{~cm} \rightarrow \mathrm{CTDI}_{\mathrm{vol}, 14}= \\
& 7.1 \mathrm{mGy}(\sigma=8.6 \mathrm{HU}) \\
- & 5-20 \mathrm{~kg} \rightarrow \text { equivalent } \varnothing \text { of } 16 \mathrm{~cm} \rightarrow \mathrm{CTDI}_{\mathrm{vol}, 16}= \\
& 9.4 \mathrm{mGy}(\sigma=9.4 \mathrm{HU}) \\
- & 20 \text { and } 35 \mathrm{~kg} \rightarrow \text { equivalent } \varnothing \text { of } 20 \mathrm{~cm} \rightarrow \mathrm{CTDI}_{\mathrm{vol}, 20}= \\
& 14.0 \mathrm{mGy}(\sigma=11.5 \mathrm{HU}) \\
- & 35 \text { and } 50 \mathrm{~kg} \rightarrow \text { equivalent } \varnothing \text { of } 24 \mathrm{~cm} \rightarrow \mathrm{CTDI}_{\mathrm{vol}, 24=}= \\
& 18.6 \mathrm{mGy}(\sigma=15.0 \mathrm{HU})
\end{aligned}
$$

From the data in Table 1 , these $\mathrm{CTDI}_{\mathrm{vol}, \varnothing}$ values can be converted into a CTDI $_{\mathrm{vol}}$ measured in a standard CTDI test object $(16$ or $32 \mathrm{~cm})$ For each weight class, 
Table 3 CT acquisition parameters for routine abdominal CT as a function of patient's weight

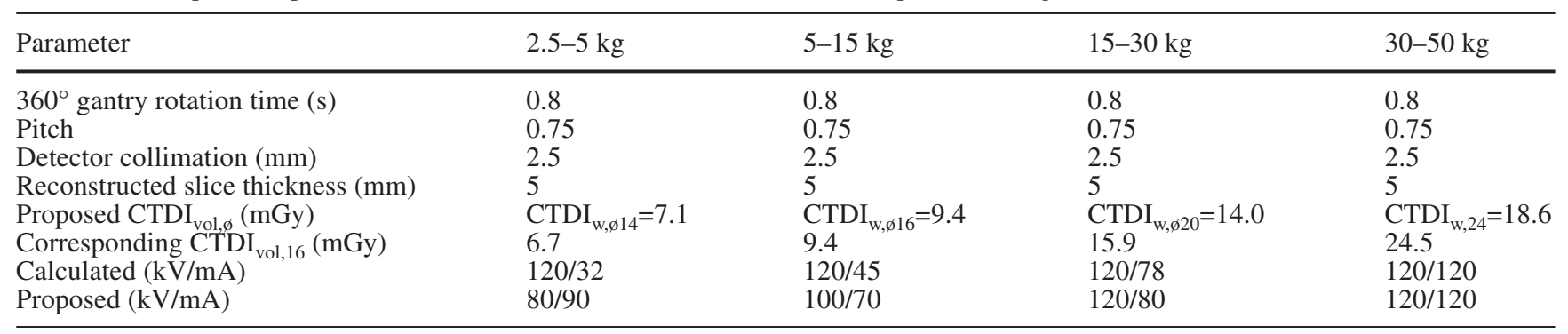

Table 4 Comparison of the $\mathrm{CTDI}_{\mathrm{vol}}$ values obtained in this study with data available in the literature

\begin{tabular}{|c|c|c|c|c|}
\hline Proposed CTDI $_{\mathrm{vol}}$ values & $2.5-5 \mathrm{~kg}$ & $5-15 \mathrm{~kg}$ & $15-30 \mathrm{~kg}$ & $30-50 \mathrm{~kg}$ \\
\hline $\mathrm{CTDI}_{\mathrm{vol}}$ (mGy) (this study) & 6.7 & 9.4 & 15.9 & 24.5 \\
\hline $\operatorname{Ref~CTDI}_{\mathrm{w}}(\mathrm{mGy})[15]^{\mathrm{a}}$ & 17 & $20-23$ & $23-28$ & $34-41$ \\
\hline $\operatorname{Ref~CTDI}_{\mathrm{w}}^{\mathrm{w}}(\mathrm{mGy})[25]$ & 20 & $20-25$ & $25-30$ & - \\
\hline Age classes & $<2$ years & $2-6$ years & 6-14 years & $14-18$ years \\
\hline $\operatorname{Ref~CTDI}_{\mathrm{w}}(\mathrm{mGy})$ [27] & 5.6 & $12-2$ & 14 & 23.5 \\
\hline
\end{tabular}

a The eff $\mathrm{CTDI}_{\mathrm{w}, 16}$ was calculated using a ${ }_{\mathrm{n}} \mathrm{CTDI}_{\mathrm{w}, 16}$ of $0.277 \mathrm{mGy} \mathrm{mAs}^{-1}$ (value measured at $140 \mathrm{kV}$ on our unit), a pitch factor of 0.75 and a $360^{\circ}$ gantry rotation time of $0.8 \mathrm{~s}$.
CTDI $_{\mathrm{vol}, 16}$ considered to give acceptable compromises between dose and image noise are given in Table 3 together with tube voltage and tube current possible combinations. From the data in Table 4 it can be observed that the CTDI ${ }_{\mathrm{vol}}$ for routine examinations using the proposed protocols is one half to one third of some of the values reported in the literature $[25,26]$ but are in good agreement with values proposed by Greess et al. [27].

None of the three radiologists involved in this study considered that the amount of quantum mottle present in the images necessitated an increase in patient dose. On the contrary, images were considered of very good quality. Thus, dose levels especially for small children can be further reduced especially when the detection of lowcontrast lesions is not of primary importance. Of the 507 examinations performed, only seven were considered inadequate for diagnosis. All of the rejected images had image artifacts due to metallic implants. The remaining 500 examinations were considered adequate for diagnosis.

\section{Discussion}

According to the results published recently by Cohnen et al. [28], an excellent correlation exists between effective dose and CTDI measurements. Thus results presented in Fig. 2 clearly demonstrate that a low level of image noise cannot be kept constant in a wide range of patient weight (or abdominal section diameter) without increasing significantly patient dose. The use of the low- contrast detection constraint chosen in this paper led to $\mathrm{CTDI}_{\mathrm{vol}}$ values ranging from $0.4 \mathrm{mGy}$ for a $16 \mathrm{~cm}$ object to $25.5 \mathrm{mGy}$ for a $32 \mathrm{~cm}$ object. If a $\mathrm{CTDI}_{\mathrm{vol}}$ of $25.5 \mathrm{mGy}$ for adults seems realistic when compared to the reference dose level of $35 \mathrm{mGy}$ proposed for abdominal examinations by the CEC guidelines [29], a CTDI ${ }_{\mathrm{vol}}$ of $0.4 \mathrm{mGy}$ for children appears unrealistic and could lead to the use of the unit in a region where it is no longer quantum noise limited. For our CT unit this would require a tube voltage of $80 \mathrm{kV}$, a tube current of $18 \mathrm{~mA}$, a $360^{\circ}$ tube rotation time of $0.5 \mathrm{~s}$ and a pitch of 1.5 . The reduction of the size of the structure to be detected by a factor of two (i.e., $d=5 \mathrm{~mm}$ ) would require $\mathrm{CTDI}_{\mathrm{vol}}$ values of 1.6 and $79.5 \mathrm{mGy}$ for $16-$ and a $32-\mathrm{cm}$ test objects, respectively. While the $\mathrm{CTDI}_{\mathrm{vol}}$ obtained for the $16-\mathrm{cm}$ object seems more realistic in term of machine parameters $(80 \mathrm{kV}, 72 \mathrm{~mA}, 0.5 \mathrm{~s}$ and a pitch of 1.5$)$, the parameters obtained for the $32-\mathrm{cm}$ object are certainly inadequate. For our CT unit it would require a tube voltage of $140 \mathrm{kV}$, a tube current of $500 \mathrm{~mA}$, a $360^{\circ}$ tube rotation time of $0.8 \mathrm{~s}$ and a pitch of 0.75 , delivering an effective dose of about $40 \mathrm{mSv}$ for a scan length of $30 \mathrm{~cm}$ [29]. This clearly demonstrates that in order to maintain a reasonable patient dose, we must accept working with various noise levels, resulting in a situation where lowcontrast detection will depend on patient size.

If we accept that images of large patients cannot offer the same level of low-contrast detection as images of thin patients, the problem remains on how to propose acceptable compromises. In this study, the dose efficacy concept was introduced and used at a level of $0.5 \mathrm{HU} \mathrm{mGy}-1$ on the basis of results obtained from the Rose model observer. It appears that for the unit and slice thickness investigated this dose efficacy leads to an image noise level of about $10 \mathrm{HU}$ for children weighing 
up to $30 \mathrm{~kg}$ and an image noise level of $15 \mathrm{HU}$ for children in the weight class $30-50 \mathrm{~kg}$. According to data published by Starck et al. [30], this corresponds to images of very good quality, since images with a standard deviation of $10 \mathrm{HU}$ were considered of high quality whereas images with a standard deviation of $30 \mathrm{HU}$ were considered very noisy. Thus, the dose levels proposed in this paper can be reduced when image quality requirements are not particularly high.

Manufacturers are developing strategies in order to adapt tube current to the anatomy of the patient. These strategies are generally based on the assessment of the dose received by the detectors and allow the control of the amount of quantum noise present in the images. On some units the user is asked to enter the image noise level (e.g., the standard deviation value) he wants to work with. This paper provides some hints for choosing these levels. At the same time, manufacturers are proposing the display of the $\mathrm{CTDI}_{\mathrm{vol}}$ corresponding to the acquisition protocol chosen by the user. This quantity integrates the pitch information and corresponds to the averaged dose received in the slice. It is thus directly linked with the amount of quantum noise present in an image. However, one has to be sure that the $\mathrm{CDTI}_{\mathrm{vol}}$ indicated by the unit is expressed in the CTDI test object of $16 \mathrm{~cm}$ in diameter when dealing with pediatric acquisition, otherwise large dose underestimation would be made.

Having defined $\mathrm{CTDI}_{\mathrm{vol}}$ for each weight class, the tube currents were calculated from the ${ }_{\mathrm{n}} \mathrm{CTDI}_{\mathrm{w}}$ measured at $120 \mathrm{kV}$ (see Table 3). Since no major beam hardening effect is expected when dealing with small abdominal diameters, a tube voltage of $80 \mathrm{kV}$ was chosen for the first weight class (i.e., $2.5-5 \mathrm{~kg}$ ) and a tube voltage of $100 \mathrm{kV}$ was chosen for the second weight class (i.e., $5-15 \mathrm{~kg}$ ). This allows a slight increase of the contrast-to-noise ratio of the acquisition and might allow us to further reduce patient dose without reducing the detection of lesions [21].

To conclude, we have shown that the CTDI test objects can be used to find reasonable compromises between image noise and dose as a function of patient weight, and that it is possible to work well below some of the $\mathrm{CTDI}_{\mathrm{vol}}$ proposed in the literature while keeping an acceptable image noise level. The "automatic exposure systems" developed by manufacturers will be of great help to control patient exposure especially when dealing with the premature or neonate population where dose efficacy is very high.

\section{References}

1. Shrimpton PC, Jones DG, Hillier MC, Wall BF, Le Heron JC, Faulkner K (1991) Survey of CT practice in the UK. Part 2: dosimetric aspects, NRPB R249. NRPB, Chilton, UK

2. Brix G, Nagel HD, Stamm G, Veit R, Lechel U, Griebel J, Galanski M (2003) Radiation exposure in multislice versus single-slice spiral CT: results of a nationwide survey. Eur Radiol 13(8):1979-1991

3. Golding SJ, Shrimpton PC (2002) Radiation dose in CT: are we meeting the challenge? Br J Radiol 75:1-4

4. Kaul A, Bauer B, Bernhardt J, Nosske D, Veit R (1998) Effective doses to members of the public from the diagnostic application of ionizing radiation in Germany. Eur Radiol 7(7):1127-1132

5. Giacomuzzi SM, Erckert B, Schopf T, Freund MC, Springer P, Dessl A, Jaschke W (1996) The smart-scan procedure of spiral computed tomography. A new method for dose reduction. Rofo Fortschr Geb Rontgenstr Neuen Bildgeb Verfahr 165(1):10-16
6. Kalender WA, Wolf H, Suess C, Gies M, Greess H, Bautz WA (1999) Dose reduction in CT by on-line tube current control: principles and validation on phantoms and cadavers. Eur Radiol 9(2):323-328

7. Greess H, Wolf $H$, Baum U, Lell M, Pirkl M, Kalender W, Bautz WA (2000) Dose reduction in computed tomography by attenuation-based on-line modulation of tube current: evaluation of six anatomical regions. Eur Radiol 10(2):391-394

8. Toth TL, Bromberg NB, Pan TS, Rabe J, Woloschek SJ, Li J, Seidenschnur GE (2000) A dose reduction $\mathrm{X}$-ray beam positioning system for high-speed multislice CT scanners. Med Phys 27(12):2659-2668

9. Kearney SE, Jones P, Meakin K, Garvey CJ (1997) CT scanning of the paranasal sinuses: the effect of reducing mAs. Br J Radiol 70:1071-1074

10. Cohnen M, Fischer H, Hamacher J, Lins E, Kotter R, Modder U (2000) CT of the head by use of reduced current and kilovoltage: relationship between image quality and dose reduction. Am J Neuroradiol 21(9):1654-1660

11. Ravenel JG, Scalzetti EM, Huda W, Garrisi W (2001) Radiation exposure and image quality in chest CT examinations. Am J Roentgenol 177:279-284
12. Kalra MK, Prasad S, Saini S, Blake MA, Varghese J, Halpern EF, Rhea JT, Thrall JH (2002) Clinical comparison of standard-dose and $50 \%$ reduceddose abdominal CT: effect on image quality. Am J Roentgenol 179:1101-1106

13. Huda W, Atherton JV, Ware DE, Cumming WA (1997) An approach for the estimation of effective radiation dose at CT in pediatric patients. Radiology 203:417-422

14. Chapple CL, Willis S, Frame J (2002) Effective dose in pediatric computed tomography. Phys Med Biol 47:107-115

15. Paterson A, Frush DP, Donnelly LF (2001) Helical CT of the body: are settings adjusted for pediatric patients? AJR Am J Roentgenol 176:297-301

16. Donnelly LF, Emery KH, Brody AS, Laor T, Gylys-Morin VM, Anton CG, Thomas SR, Frush DP (2001) Minimizing radiation dose for pediatric body, application of single-detector helical CT: strategies at a large children's hospital. AJR Am J Roentgenol 176:303-306 
17. Wormanns D, Diederich S, Lenzen H, Lange P, Link TM, Ludwig K, Papke K, Hagedorn C, Heindel W (2001) Abdominal spiral CT in children: which radiation exposure is required? Eur Radiol 11(11):2262-2266

18. Christoph Suess C, Chen X (2002) Dose optimization in pediatric CT: current technology and future innovations. Pediatr Radiol 32:729-734

19. Huda W, Scalzetti EM, Levin G (2000) Technique factors and image quality as function of patient weight at abdominal CT. Radiology 217:430-435

20. Huda W (2002) Dose and image quality in CT. Pediatr Radiol 32:709-713

21. Boone JM, Geraghty EM, Seibert JA, Wootton-Gorges SL (2003) Dose reduction in pediatric $\mathrm{CT}$ : a rational approach. Radiology 228(2):352-360
22. International Electrotechnical Committee (1999) Medical diagnostic $\mathrm{X}$-ray equipment-particular requirements for the safety of X-ray equipment for CT; Standard IEC \# 60601-2-44

23. International Electrotechnical Committee (2001) Medical diagnostic $\mathrm{X}$-ray equipment-particular requirements for the safety of X-ray equipment for CT; Standard IEC \# 60601-2-44 amendment 1

24. Boone JM, Nelson TR, Lindfors KK, Seibert JA (2001) Dedicated breast CT: radiation dose and image quality evaluation. Radiology 221:657-667

25. Shrimpton PC, Wall BF (2000) Reference doses for pediatric computed tomography. Radiat Protect Dosim 90(1-2):249-252

26. Frush DP, Soden B, Frush KS, Lowry C (2002) Improved pediatric multidetector body CT using a size-based color-coded format. Am J Roentgenol 178:721-726
27. Greess H, Nömayr A, Wolf H, Baum U, Lell M, Böwing B, Kalender W, Bautz WA (2002) Dose reduction in CT examination of children by an attenuation-based on-line modulation of tube current (CARE Dose). Eur Radiol 12:1571-1576

28. Cohnen M, Poll LJ, Puettmann C, Ewen K, Saleh A, Modder U (2003) Effective doses in standard protocoles for multi-slice CT scanning. Eur Radiol 13(5):1148-1153

29. Commission of the European Communities (2000) European guidelines on quality criteria for computed tomography, Luxembourg EUR $16262 \mathrm{EN}$

30. Starck G, Lönn L, Cederblad Å, Forsell-Aronsson E, Sjöström L, Alpsten M (2002) A method to obtain the same levels of CT image noise for patients of various sizes, to minimize radiation dose. Br J Radiol 75:140-150 